\section{HASTA LA VISTA, NEEDLE}

The Sharps Terminator is a new device that destroys syringe needles immediately after use, rendering them harmless. It destroys the needle shaft in a single-handed action leaving the user with only the plastic syringe. It has the potential to significantly reduce the number of needlestick injuries reported by healthcare workers every year in the UK.

The Sharps Terminator ensures that healthcare providers are able to implement safe procedures for using and disposing of sharp medical instruments and contaminated waste; provide sharps disposal equipment as close as possible to the assessed areas where they are used; and prevent the need to recap the needle.

The Sharps Terminator is lightweight, portable and battery-powered. Once the needle is withdrawn from the patient, the needle is simply inserted into the Terminator and held for three seconds. As the needle body completes a circuit between two angled copper electrodes, resistance from the needle creates sufficient heat to destroy the metal portion of the needle from the tip to the hub. The Terminator then cuts the plastic 'hub' above the needle so that no metal is left on the syringe. The debris from the needle falls into a clean-out tube at the base of the device where it is exposed to UV light to eliminate contaminants.

The Sharps Terminator is distributed by VLA Healthcare: telephone 08454531377 or email info@vla-healthcare.com.

\section{HARNESS THE POWER OF OZONE}

Biowell is committed to developing ozonated water disinfection products to improve the quality of patient care and hygiene in dentistry. Using Biowell ozonated water demonstrates a conscious choice to raise safety assurance, disinfection standards and water quality while remaining environmentally sustainable.

Biowell's CDU Compact Disinfection Unit offers all-round protection in the dental practice: greater sanitation via hand-wash and instrument pre-wash; a bacteria-free water source; oral applications such as mouth rinse and irrigation of the treatment area; and surface disinfection of dental chairs, equipment, countertops, cabinets, handles and office surfaces.

The CDS Central Disinfection System is a point-of-use ozone treatment to purify the water supply. Ozone acts on contact to eliminate 99.999\% of all bacteria, including legionella, viruses and moulds and prevents biofilm and slime from forming. It exhibits a short half-life before reverting back to oxygen and provides a natural residual disinfection pipe; water line sanitation is maintained without affecting taste or smell.

Biowell also offer a spray bottle with special coating to preserve the antibacterial properties of ozonated water. This product enables you to use ozone's quick disinfecting and deodorising abilities on the go.

To find out more and to order visit http://biowellozone.com, telephone 03301239403 or emailinfo@biowellozone.com.

\section{REVOLUTIONARY TRIANGLE FULL ARCH FOV}

The latest Morita cone beam CT scanner, the Veraviewepocs 3D R100, won the first Innovations Award at the Clinical Innovations Conference in London. The prize showcases the most pioneering products on the dental market. The Veraviewepocs 3D R100 received the award for its revolutionary Reuleaux triangle full arch field of view. By closely matching the natural dental arch, patient X-ray dosage is reduced by around 15\%, compared with a conventional $100 \mathrm{~mm}$ cylindrical scan.

The Morita Veraviewepocs 3D R100 captures posterior dentition that might be missed by a conventional $80 \mathrm{~mm}$ cylindrical FOV scanner. The unique triangular field of view excludes anatomy outside the jawline which would be exposed to radiation using a standard $100 \mathrm{~mm}$ diameter cylindrical FOV. The enlarged field of view is especially important when planning implant cases requiring surgical guides and full arch restoration.

With up to three times the image detail of other 3D $\mathrm{X}$-ray systems, the Morita Veraviewepocs 3D R100 and F40 offer the best value in terms of resolution and capital cost. In the UK they are exclusively available from The Dental Imaging Company.

For a free demonstration of the Morita Veraviewepocs 3D R100 or F40, call 08453883380 or email:info@morita-uk.com.

\section{DESIGNER DENTAL CABINETRY}

RPA Dental prides itself on offering clients exceptional levels of customer service and support as well as products from some of the world's leading manufacturers. Among their foremost product lines is the Tavom range of designer dental cabinetry.

Tavom cabinetry is manufactured to the highest of clinical standards both for surgery and decontamination environments. Each of Tavom's 7,000 modular elements comes in a range of five different styles with over 350 colour combinations available, including a variety of worktop materials.

To discuss your requirements or for a little advice from the experts, call RPA Dental on 08000933 975, or visit their new equipment website www.rpadental.net. 